\title{
Analysis and Modeling of Capacitive Power Transfer in Microsystems
}

\author{
Mustafa Emre Karagozler, Student Member, IEEE, Seth C. Goldstein, Senior Member, IEEE and \\ David S. Ricketts, Member, IEEE
}

\begin{abstract}
As externally powered microsystems become more common, designers need better tools to understand power delivery systems such as non-resonant capacitive coupling. In this paper we present the first general method which allows a designer to easily model power delivery through capacitive coupling. The method uses a power iteration technique which allows one to analyze systems when the time to charge the coupling capacitor is longer than a charge cycle, enabling us to analyze a greater range of systems than previously possible. In fact, we are able to model the entire system with an equivalent resistance.

We show that our model accurately reproduces both static and dynamic characteristics of the exact solution and that this model is general, in that it is valid for capacitor charge times that are longer as well as shorter than a charge cycle. This model also reveals several regions of operation where different parameters (e.g. capacitance, frequency and series resistance) dominate, allowing the designer to quickly and intuitively understand the design space for capacitive power transfer.
\end{abstract}

Index Terms-Capacitive coupling, power transfer, power conversion, energy harvesting.

\section{INTRODUCTION}

Externally powered microsystems are of increasing interest for a wide range of applications, such as biomedical implants [1], energy scavenging sensors [2] and micro-scale robotics [3]. One common power delivery method is capacitive coupling an $a c$ source across an isolating interface and then rectifying the $a c$ signal to a $d c$ voltage on a local (in-system) storage capacitor. In many of these applications, the large thickness and the low dielectric constant of the interface material result in a very small coupling capacitance [1]. In particular, this coupling capacitance is often much less than the storage capacitance that supplies the system. The result is that only a small amount of charge is delivered per cycle and it takes a large number of charge cycles to charge the local capacitor. Moreover, due to the finite resistance of a rectifying stage and high frequency of the input signal, the time to fully refresh the coupling capacitors, characterized by the time constant $\tau$, can be much larger than the period of a charge cycle, $t_{0}$, resulting in each cycle only partially charging the capacitors, i.e. the system does not reach steady state during a charge cycle. This presents a particular challenge to the designer, as previous analysis of rectifying circuits have

Karagozler and Ricketts at Electrical and Computer Engineering at Carnegie Mellon University, 5000 Forbes Ave, Pittsburgh, PA 15213 \{mkaragoz, ricketts\}eece.cmu.edu. Goldstein in Computer Science Department at Carnegie Mellon University sethecs. cmu.edu.

Copyright (C) 2011 IEEE. Personal use of this material is permitted. However, permission to use this material for any other purposes must be obtained from the IEEE by sending an email to pubs-permissions@ieee.org.

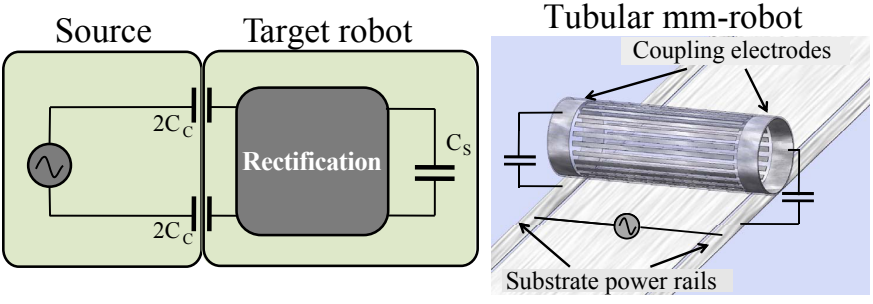

(a)

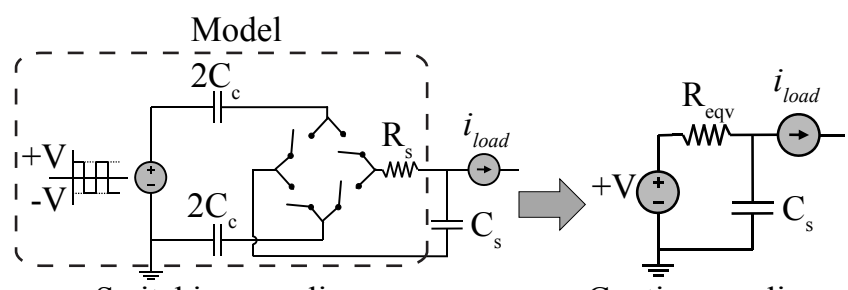

Switching; nonlinear

Continuous; linear

(b)

Fig. 1. (a) Power transfer to/between robots. $C_{c}$ : coupling capacitor; $C_{s}$ : storage capacitor. (b) Modeling goal of rectifier and coupling capacitor power transfer.

used a switched capacitor approach, where they have assumed that the capacitors reach steady state or are fully charged at the end of each cycle [4], [5], [6], i.e. $\tau \ll t_{0}$.

In this work, we analyze capacitive power transfer for general $\tau$ through a novel approach using a power iteration method. This enables us to find an analytical relationship between: the coupling and storage capacitors, the finite resistance of the rectifier and the charge period or frequency. We focus our analysis when $\tau$ is comparable or greater than $t_{0}$, as the simple case when $\tau$ is less than $t_{0}$ can be solved with known techniques (See Appendix A).

We use this analysis to derive an equivalent resistance, $R_{e q v}$, which allows one to easily model the entire power transfer system with a single linear component. The analytical framework enables one to see the dependance of $R_{\text {eqv }}$ on the underlying circuit elements. In addition we show that the model is general for all $\tau$. The model also shows that there is a critical frequency, $f_{\text {knee }}$, below which $R_{\text {eqv }}$ is dominated by the rectifier dynamics and not the series resistance of the rectifier and above which $R_{e q v}$ reduces to the series resistance $R_{s}$, indicating that the rectifier dynamics are not a limiting factor in charge delivery. The model details the resulting performance and cross-over between these two operating regions. 
In addition we show the inter-dependence of the coupling and storage capacitor ratio on charge time through the $R_{e q v}$ that is developed.

With the analytical model developed in this paper the designer is able to analyze the performance of a proposed capacitive power transfer system with respect to key circuit components, such as the coupling capacitor and series resistance as well as desired operation frequency, without the need to perform exhaustive SPICE simulations for each case.

The paper is organized as follows. Section II outlines the goals and approach of our analysis and model. Section III analyzes in detail the charge transfer in a capacitively coupled rectifier circuit. Section IV uses these results to model accurately both steady-state and dynamic response of the power delivery circuit, including an analysis of energy transfer and power dissipated in the system. Section V summarizes the findings of this work. The appendices contain detailed calculations of key steps used throughout the paper.

\section{Power Transfer Model}

Our general system is modeled by an external ac source, capacitively coupled to a microsystem, such as a microrobot [7], Fig. 1(a). Charge is delivered through the capacitive link and then rectified by an in-system rectifier and stored on a local storage capacitor, $C_{s}$. While the canonical rectifier uses diodes, many systems enhance efficiency using a synchronous FET rectifier [8], [9]. The function of the two is the same: they allow charge to flow through one path during the positive $a c$ cycle and through another path during the negative cycle.

Our approach is to model the bridge rectifier as a switch element (diode or FET) and a series resistance, Fig. 2(a). For a diode bridge, we analyze the non-ideal diodes using their linearized models as shown in Fig. 2(b). We group the series resistance of the diodes with the resistance of rest of the circuit, $R_{c}$, creating a single lumped resistance of $R_{s}=R_{c}+2 R_{O N}$.

For any excitation frequency, $f$, of the source, applying a square wave-instead of a sine wave, for example-is the fastest way of charging the storage capacitor on the target robot, since it provides the largest voltage drop at any time. For this reason, a square wave generator is analyzed as the voltage source on the source robot. The generated voltage alternates between $+V$ and $-V$, with a period of $2 t_{0}$. Figure 3 shows the sequence of each transition. A single cycle of the source is split into two parts, an odd and an even half-half cycle, where $t_{0}$ is the duration of the half cycle and $n$ represents the $n^{t h}$ half cycle $\left(n \in Z^{+}\right)$. The continuous voltage waveform on the storage capacitor is $V_{C_{s}}(t)$ and the discrete voltage waveforms on the storage capacitor are $V_{C_{s}, n}$ and $V_{C_{s}, m}$, where $n$ is the discrete voltage series at every half cycle and $m$ is the discrete voltage series at every cycle $(m=2 n)^{1}$. The discrete voltages

\footnotetext{
${ }^{1}$ For systems that charge quickly, $V_{C_{s}}(t)$ is significantly different in each half cycle and it is necessary to consider each half cycle for an accurate representation. For slowly charging systems, i.e. $C_{s} \gg C_{c}, V_{C_{s}}(t)$ is approximately symmetric for each half cycle, such that the whole cycle can be approximated by two symmetric half cycles, and the series represented by only the cycle transitions, $m$.
}

are defined at the voltage $V_{C_{s}}\left(n t_{0}^{-}\right)$, where $t_{0}^{-}$is the instant just before the start of the next half cycle.

Our goal is to develop a simple model for capacitive power transfer, Fig. 1(b), when $\tau \gg t_{0}$ that replaces the switching source, coupling capacitors and nonlinear bridge with a continuous time, linear $R_{e q v}$. To do this, we:

1) Calculate the voltage on the storage capacitor, $V_{C_{s} n}$, at discrete times, $m t_{0}$.

2) Calculate the voltage across the coupling capacitor and rectifier, $\Delta V=V-V_{C_{s} n}$.

3) Calculate the charge, $Q_{n}$, transferred into the storage capacitor in one cycle.

4) Calculate the average current, $i_{a v g}$, by dividing the current delivered in one cycle by the time of that cycle, $t_{0}$.

5) Determine that the ratio $\Delta V / i_{a v g}$ is independent of time and input/output voltages.

6) Model the $I-V$ characteristics as $R_{e q v}=\Delta V / i_{a v g}$, which may be a function of the circuit parameters: $C_{c}$, $C_{s}, f$ (or $t_{0}$ ), and $R_{s}$.

With $R_{e q v}$, one can determine and optimize:

- Storage capacitor charge and discharge time

- Static and transient voltage drop due to current loads, $i_{\text {load }}$

- Power dissipation and transfer efficiency

analytically, allowing the designer to explore the design space easily, without the need for exhaustive simulations of all possible cases.

\section{Time Analysis of Power Transfer Circuit}

There are two cases, $C_{c}>C_{s}$ and $C_{c}<C_{s}$, and two parameter spaces, $\tau<t_{0}$ and $\tau \geq t_{0}$ that one may consider in capacitive power transfer. The first case is when $C_{c} \geq C_{s}$ with either $\tau>t_{0}$ or $\tau<t_{0}$; the charge cycle requires only a few cycles to charge and may be modeled with a capacitive divider. The second case is when $C_{c} \ll C_{s}$ with $\tau \ll t_{0}$ or $\tau \geq t_{0}$. The former can be analyzed, using a switch capacitor approach [4], [5], [6], where it is assumed the capacitors charge each cycle; a brief derivation is included in Appendix A for completeness of this analysis. The later case, $\tau \geq t_{0}$, requires a new approach that models the dynamics of the capacitor voltage during each charge cycle. We will show that through such a new approach, we are able to develop a generalized analytical model for capacitive power transfer that is valid for all $\tau$.

\section{A. Governing Equations}

We begin by solving the voltage, $V_{C_{s} n}$. We assume that the source voltage starts with a positive cycle, where the source voltage is $+V$, as in Fig. 3(a). Initially, at time $t=0^{-}$, neither the coupling capacitors nor the storage capacitor are charged. At $t=0^{+}$, the voltage across the source becomes $+V$, and both capacitors begin to charge. We first examine the continuous time solution for each cycle and then discretize the continuous solution at the end of this subsection.

Kirchhoff's voltage law for the circuit, during $0<t<t_{0}$ or $n=$ odd cycle, can be written as:

$$
V-V_{C_{c}}(t)-V_{C_{s}}(t)-i(t) R_{s}=0,
$$




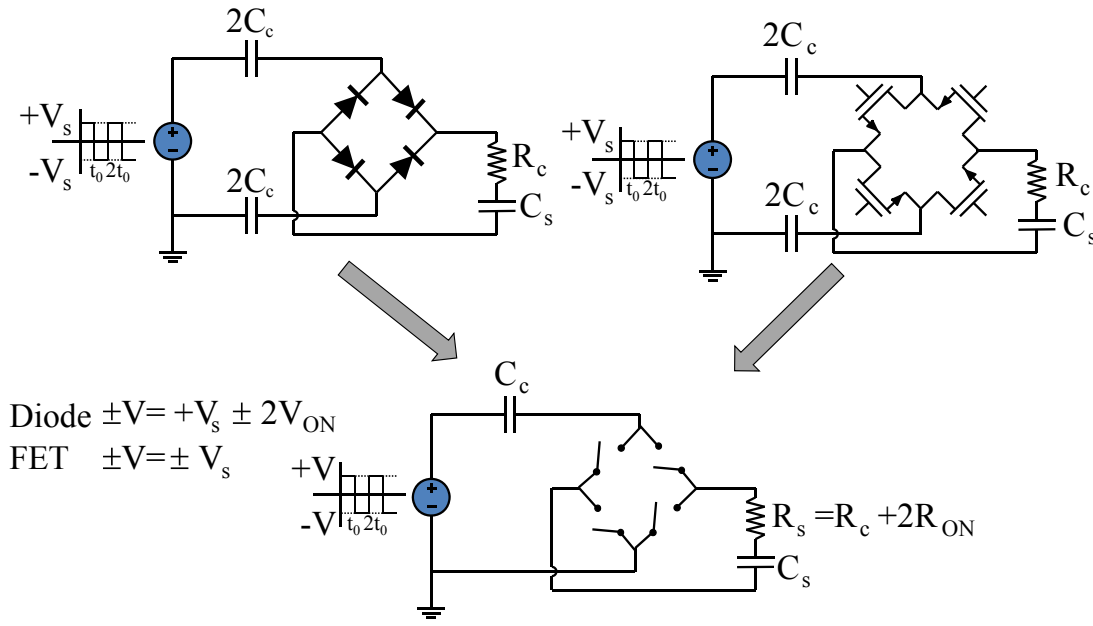

(a)

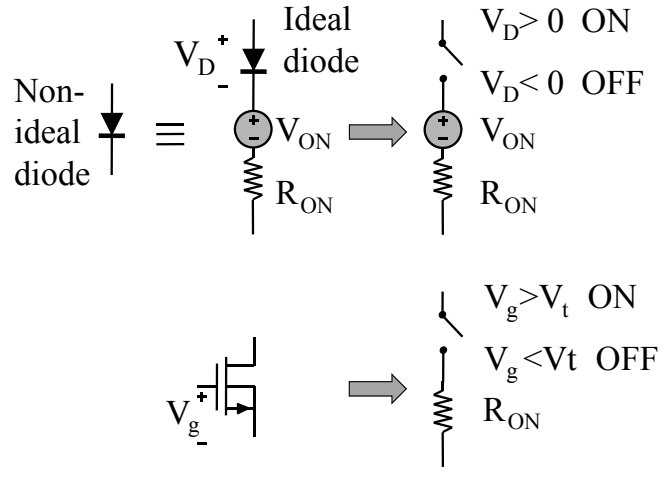

(b)

Fig. 2. (a) Diode and FET model. (b) Diode and FET bridges reduce to same circuit model. $V_{O N}$ of diode is subtracted from source voltage to produce an equivalent drive voltage for the general model.

which is a function of three unknowns: $V_{C_{c}}(t), V_{C_{s}}(t)$ and $i(t)$. To solve this in terms of one unknown, we will need two additional equations. The first is the charge conservation equation for the capacitors:

$$
C_{s} V_{C_{s}}(t)-C_{c} V_{C_{c}}(t)=C_{s} V_{C_{s}}\left(0^{-}\right)-C_{c} V_{C_{c}}\left(0^{-}\right) .
$$

Notice that for the first positive cycle, $V_{C_{s}}\left(0^{-}\right)=V_{C_{c}}\left(0^{-}\right)=$ 0 , as the capacitors start off discharged. The second relation is the current through the capacitors (the current is defined to be positive flowing into the "+" terminal of the capacitors):

$$
i(t)=C_{s} \frac{\mathrm{d} V_{C_{s}}(t)}{\mathrm{d} t}=C_{c} \frac{\mathrm{d} V_{C_{c}}(t)}{\mathrm{d} t} .
$$

With these, we can write (1) in terms of $V_{C_{s}}(t)$ or $V_{C_{c}}(t)$ :

$$
\begin{array}{r}
R C_{s} \frac{\mathrm{d} V_{C_{s}}(t)}{\mathrm{d} t}=\left(V+\frac{C_{s}}{C_{c}} V_{C_{s}}\left(0^{-}\right)-V_{C_{c}}\left(0^{-}\right)\right) \\
-V_{C_{s}}(t)\left(\frac{C_{s}}{C_{c}}+1\right), \\
R C_{c} \frac{\mathrm{d} V_{C_{c}}(t)}{\mathrm{d} t}=\left(V+\frac{C_{c}}{C_{s}} V_{C_{c}}\left(0^{-}\right)-V_{C_{s}}\left(0^{-}\right)\right) \\
-V_{C_{c}}(t)\left(\frac{C_{c}}{C_{s}}+1\right) .
\end{array}
$$

The solutions for $V_{C_{s}}(t)$ and $V_{C_{c}}(t)$ can be written as:

$$
\begin{aligned}
& V_{C_{s}}(t)=a(t) V+(1-a(t)) V_{C_{s}}\left(0^{-}\right)-a(t) V_{C_{c}}\left(0^{-}\right), \\
& V_{C_{c}}(t)=b(t) V-b(t) V_{C_{s}}\left(0^{-}\right)+(1-b(t)) V_{C_{c}}\left(0^{-}\right),
\end{aligned}
$$

where,

$$
\begin{aligned}
a(t) & =\frac{C_{c}}{C_{c}+C_{s}}\left(1-e^{-t / \tau}\right), \\
b(t) & =\frac{C_{s}}{C_{c}+C_{s}}\left(1-e^{-t / \tau}\right), \\
\tau & =R_{s} \frac{C_{c} C_{s}}{C_{c}+C_{s}} .
\end{aligned}
$$

At $t=t_{0}^{+}$the source voltage switches to $-V$. We follow the same procedure as before. During this cycle when $t_{0}<$ $t<2 t_{0}$ or the $n=$ even cycle, Kirchhoff's voltage equation is:

$$
-V-V_{C_{c}}(t)+V_{C_{s}}(t)-i(t) R_{s}=0 .
$$

The charge conservation equation is:

$$
C_{s} V_{C_{s}}(t)+C_{c} V_{C_{c}}(t)=C_{s} V_{C_{s}}\left(t_{0}^{-}\right)+C_{c} V_{C_{c}}\left(t_{0}^{-}\right),
$$

where we have defined the initial voltages of each cycle as the voltage just before the start of the next cycle, i.e. $t=t_{0}^{-}$, Fig. 3(b). The current through the capacitors is ${ }^{2}$ :

$$
i(t)=-C_{s} \frac{\mathrm{d} V_{C_{s}}(t)}{\mathrm{d} t}=C_{c} \frac{\mathrm{d} V_{C_{c}}(t)}{\mathrm{d} t} .
$$

The solutions for $V_{C_{s}}(t)$ and $V_{C_{c}}(t)$ are then:

$$
\begin{array}{r}
V_{C_{s}}(t)=a\left(t-t_{0}\right) V+\left(1-a\left(t-t_{0}\right)\right) V_{C_{s}}\left(t_{0}^{-}\right) \\
+a\left(t-t_{0}\right) V_{C_{c}}\left(t_{0}^{-}\right), \\
V_{C_{c}}(t)=-b\left(t-t_{0}\right) V+b\left(t-t_{0}\right) V_{C_{s}}\left(t_{0}^{-}\right) \\
+\left(1-b\left(t-t_{0}\right)\right) V_{C_{c}}\left(t_{0}^{-}\right) .
\end{array}
$$

The constants $a(t)$ and $b(t)$ are as defined in (8) and (9). It is important to note that (14) and (15) are coupled and dependent on, $R_{s}, C_{c}$, and $C_{s}$ through the coefficients $a(t)$ and $b(t)$. When $\tau \ll t_{0}$ they can be decoupled using (1) by setting the $i\left(t_{0}^{-}\right) R_{s}$ term to zero - this is equivalent to assuming the capacitors are fully charged at the end of each cycle - and the solution derived using standard switched capacitor approaches [4], [5], [6]. In the generalized case we analyze here, without the assumption that $\tau \ll t_{0}$, (14) and (15) cannot simply be decoupled and a different analytical method is needed.

We discretize the continuous time problem by replacing $t$ with $n t_{0}^{-}$, which samples the continuous time solution just

\footnotetext{
${ }^{2}$ The negative sign is a result of the current flowing into the "-" terminal of $C_{s}$.
} 


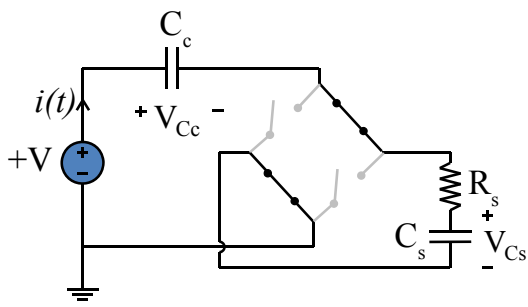

Positive cycle, $n=$ odd

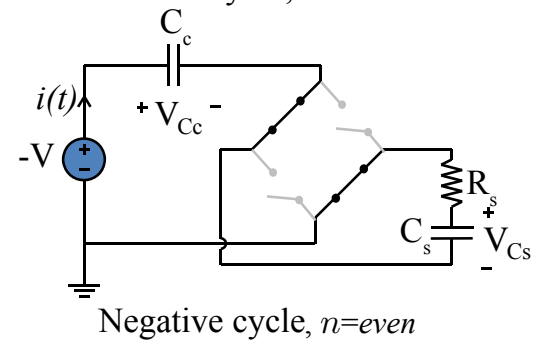

(a)

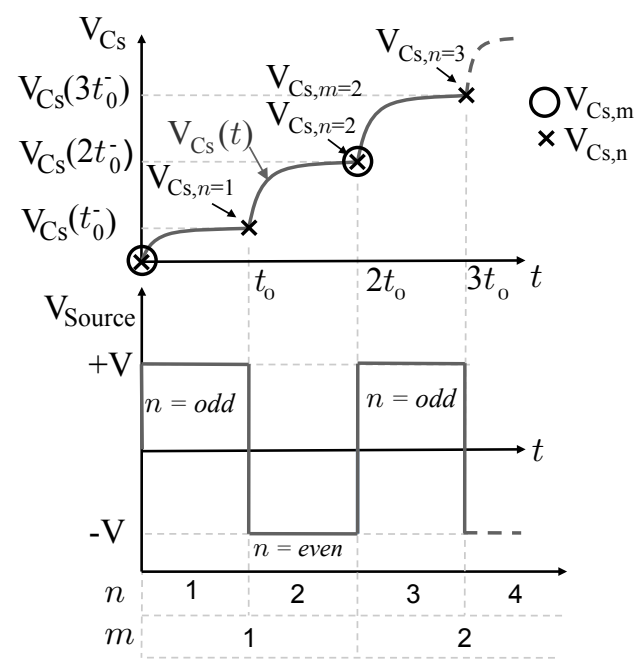

(b)

Fig. 3. The effective circuit during positive and negative source cycles when the source voltage is $+V$ and $-V$, respectively.

prior to the transition to the next cycle [See Fig. 3(b)]. We will solve (6), (7), (14) and (15) for the capacitor voltages as algebraic series of $n t_{0}^{-}$:

$$
V_{C_{s} n}=V_{C_{s}}\left(n t_{0}^{-}\right),
$$

and,

$$
V_{C_{c} n}=V_{C_{c}}\left(n t_{0}^{-}\right) .
$$

We now rewrite (6), (7), (14) and (15) in discrete time using $V_{C_{s} n}=V_{C_{s}}\left(n t_{0}^{-}\right)$and substitute the constants $a=a\left(t_{0}\right)$ and $b=b\left(t_{0}\right)$ for $a(t)$ and $b(t)$. For the $n$ is odd case:

$$
\begin{aligned}
& V_{C_{s} n}=a V+(1-a) V_{C_{s}(n-1)}-a V_{C_{c}(n-1)}, \\
& V_{C_{c} n}=b V-b V_{C_{s}(n-1)}+(1-b) V_{C_{c}(n-1)} .
\end{aligned}
$$

For $n$ is even case:

$$
\begin{aligned}
& V_{C_{s} n}=a V+(1-a) V_{C_{s}(n-1)}+a V_{C_{c}(n-1)}, \\
& V_{C_{c} n}=-b V+b V_{C_{s}(n-1)}+(1-b) V_{C_{c}(n-1)} .
\end{aligned}
$$

As mentioned above these equations are coupled and thus it is not possible derive an equation for either $V_{C_{s} n}$ or $V_{C_{c} n}$ independent of the other. To overcome this problem, we construct a system representation of the coupled variables by defining a vector $X_{n}^{3}$ :

$$
X_{n}=\left[\begin{array}{l}
V_{C_{s} n} \\
V_{C_{c} n}
\end{array}\right] .
$$

Then, for $n$ is odd case:

$$
X_{n}=\left[\begin{array}{cc}
(1-a) & -a \\
-b & (1-b)
\end{array}\right] X_{(n-1)}+V\left[\begin{array}{l}
a \\
b
\end{array}\right] .
$$

Defining $P$ and $Q$ :

$$
\begin{gathered}
P=\left[\begin{array}{cc}
(1-a) & -a \\
-b & (1-b)
\end{array}\right], \quad Q=V\left[\begin{array}{l}
a \\
b
\end{array}\right] . \\
X_{n}=P X_{(n-1)}+Q .
\end{gathered}
$$

For $n$ is even case:

$$
X_{n}=\left[\begin{array}{cc}
(1-a) & a \\
b & (1-b)
\end{array}\right] X_{(n-1)}+V\left[\begin{array}{c}
a \\
-b
\end{array}\right] .
$$

Defining $M$ and $N$ :

$$
\begin{gathered}
M=\left[\begin{array}{cc}
(1-a) & a \\
b & (1-b)
\end{array}\right], \quad N=V\left[\begin{array}{c}
a \\
-b
\end{array}\right] . \\
X_{n}=M X_{(n-1)}+N .
\end{gathered}
$$

Combining (25) and (28) we obtain (for $n$ is even),

$$
X_{n}=M P X_{(n-2)}+(M Q+N) .
$$

We have constructed an equation that relates $X_{n}$ to $X_{(n-2)}$. This is the result of combining the equations that correspond to odd and even cycles. It is important to note that (29) is for even values of $n$; i.e., a full period begins with an odd cycle and ends with an even cycle. To simplify the notation, we introduce a new subscript that accounts for the combination of the odd and even cycles: $m, m \in Z^{+}$; which represents the number of full periods. This allows us to drop explicit references to the odd and even cycles and instead represent the system in relation to a full period. We define a new vector $Y_{m}$ as:

$$
Y_{m}=X_{2 n}=\left[\begin{array}{l}
V_{C_{s} 2 n} \\
V_{C_{c} 2 n}
\end{array}\right],
$$

Thus, (29) becomes:

$$
Y_{m}=R Y_{(m-1)}+W
$$

where

$$
\begin{gathered}
R=M P=\left[\begin{array}{cc}
(1-a)^{2}-a b & a^{2}-a b \\
b^{2}-a b & (1-b)^{2}-a b
\end{array}\right], \\
W=M Q+N=\left[\begin{array}{c}
a(2-a+b) V \\
(a-b) b V
\end{array}\right] .
\end{gathered}
$$

It is possible to expand $Y_{m}$ in (31) and write it in terms of $R, W, Y_{0}=X_{0}$ and a $2 \times 2$ identity matrix $I$ :

$$
Y_{m}=\left(I+R+R^{2}+\ldots+R^{m-1}\right) W+R^{m} Y_{0} .
$$

${ }^{3} \mathrm{We}$ will use lowercase to represent scalars and uppercase to represent vectors, with the exceptions of the common usage of $R, C$, and $V$, as uppercase notation for the scalars resistance, capacitance and voltage. 
The geometric series summation in (34) can be simplified also, we know that $V_{C_{s} 0}=0$ and $V_{C_{c} 0}=0$, so $Y_{0}=0$, resulting in:

$$
Y_{m}=(I-R)^{-1}\left(I-R^{m}\right) W .
$$

In our analysis, we are mainly interested in $V_{C_{s} m}$, the voltage of the storage capacitor. We multiply from the left both sides of (35) with $\left[\begin{array}{ll}1 & 0\end{array}\right]$ to get $V_{C_{s} m}$ :

$$
\left[\begin{array}{ll}
1 & 0
\end{array}\right] Y_{m}=\left[\begin{array}{ll}
1 & 0
\end{array}\right](I-R)^{-1}\left(I-R^{m}\right) W .
$$

The left hand side of equation (36) is simply the storage capacitor voltage, $V_{C_{s} m}$. We define $A$ as:

$$
A=\left[\begin{array}{ll}
1 & 0
\end{array}\right](I-R)^{-1}=\left[\begin{array}{ll}
\frac{2+a-b}{4 a} & \frac{a-b}{4 b}
\end{array}\right] .
$$

Then, (36) becomes:

$$
V_{C_{s} m}=A W-A R^{m} W .
$$

The scalar $A W$ is computed as:

$$
A W=\left[\begin{array}{ll}
\frac{2+a-b}{4 a} & \frac{a-b}{4 b}
\end{array}\right]\left[\begin{array}{c}
a(2-a+b) V \\
(a-b) b V
\end{array}\right]=V .
$$

Then, (38) becomes:

$$
V_{C_{s} m}=V-A R^{m} W,
$$

or

$$
V_{C_{s} m}=V-\Delta V_{m}
$$

where

$$
\Delta V_{m}=A R^{m} W
$$

\section{B. Power iteration method}

To calculate $\Delta V_{m}$, which is a scalar, the matrix $R$ is raised to the power $m$, and then multiplied, from the left and the right with $A$ and $W$, respectively.

It is desirable to find a scalar expression for $\Delta V_{m}$ for easy computation. This would allow us to write (40) as a simple function of $m$, which would then allow us to calculate $V_{C_{s} m}$ analytically and also allow for the easy inversion of (41) to find $m$ for a given target $V_{C_{s} m}$, i.e. the charge time $\left(m t_{0}\right)$ of the storage capacitor. To do this, we define the eigenvectors of $R$ as $P_{1}$ and $P_{2}$ and the corresponding eigenvalues as $\lambda_{1}$ and $\lambda_{2}$, respectively (see Appendix B). By definition, when a matrix is applied to one of its eigenvectors, the result is simply the eigenvector scaled by the corresponding eigenvalue:

$$
\begin{aligned}
& R P_{1}=\lambda_{1} P_{1}, \\
& R P_{2}=\lambda_{2} P_{2} .
\end{aligned}
$$

When the $R$ matrix is raised to the power $m$, this yields:

$$
\begin{aligned}
& R^{m} P_{1}=\lambda_{1}^{m} P_{1}, \\
& R^{m} P_{2}=\lambda_{2}^{m} P_{2} .
\end{aligned}
$$

thus the power of $R$ is easily reduced to the power of a scalar, $\lambda$. To represent the right hand side of (42) in terms of scalars, we begin by writing $W$ as a linear combination of the eigenvectors of $R, P_{1}$ and $P_{2}$ :

$$
W=\alpha_{1} P_{1}+\alpha_{2} P_{2} .
$$

\begin{tabular}{|l|c||c|c||c|c|c|}
\hline \multicolumn{7}{|c|}{$C_{s}=100 \mathrm{pF}, R_{s}=100 \Omega, \kappa=0.90$} \\
\hline$C_{c} / C_{s}$ & $t_{0} / \tau$ & $C_{c}$ & $\tau$ & $\lambda_{1}$ & $\lambda_{2}$ & $\left|k_{1} / k_{2}\right|$ \\
\hline 0.001 & 5 & $1 \mathrm{e}-13$ & $9.99 \mathrm{e}-12$ & 0.996 & $4.56 \mathrm{e}-05$ & $1.03 \mathrm{e}+03$ \\
0.01 & 5 & $1 \mathrm{e}-12$ & $9.90 \mathrm{e}-11$ & 0.961 & $4.72 \mathrm{e}-05$ & $1.03 \mathrm{e}+02$ \\
0.1 & 5 & $1 \mathrm{e}-11$ & $9.09 \mathrm{e}-10$ & 0.674 & $6.74 \mathrm{e}-05$ & $1.03 \mathrm{e}+01$ \\
0.5 & 5 & $5 \mathrm{e}-11$ & $3.33 \mathrm{e}-09$ & 0.123 & $3.70 \mathrm{e}-04$ & $2.16 \mathrm{e}+00$ \\
0.9 & 5 & $9 \mathrm{e}-11$ & $4.74 \mathrm{e}-09$ & 0.013 & $3.60 \mathrm{e}-03$ & $1.41 \mathrm{e}+00$ \\
0.99 & 5 & $9.9 \mathrm{e}-11$ & $4.97 \mathrm{e}-09$ & 0.007 & $6.34 \mathrm{e}-03$ & $1.39 \mathrm{e}+00$ \\
\hline 0.001 & 1 & $1 \mathrm{e}-13$ & $9.99 \mathrm{e}-12$ & 0.998 & $1.36 \mathrm{e}-01$ & $4.69 \mathrm{e}+03$ \\
0.01 & 1 & $1 \mathrm{e}-12$ & $9.90 \mathrm{e}-11$ & 0.982 & $1.38 \mathrm{e}-01$ & $4.76 \mathrm{e}+02$ \\
0.1 & 1 & $1 \mathrm{e}-11$ & $9.09 \mathrm{e}-10$ & 0.843 & $1.61 \mathrm{e}-01$ & $5.46 \mathrm{e}+01$ \\
0.5 & 1 & $5 \mathrm{e}-11$ & $3.33 \mathrm{e}-09$ & 0.520 & $2.60 \mathrm{e}-01$ & $1.90 \mathrm{e}+01$ \\
0.9 & 1 & $9 \mathrm{e}-11$ & $4.74 \mathrm{e}-09$ & 0.389 & $3.48 \mathrm{e}-01$ & $1.67 \mathrm{e}+01$ \\
0.99 & 1 & $9.9 \mathrm{e}-11$ & $4.97 \mathrm{e}-09$ & 0.370 & $3.66 \mathrm{e}-01$ & $1.67 \mathrm{e}+01$ \\
\hline 0.001 & 0.1 & $1 \mathrm{e}-13$ & $9.99 \mathrm{e}-12$ & 1.000 & $8.19 \mathrm{e}-01$ & $4.01 \mathrm{e}+05$ \\
0.01 & 0.1 & $1 \mathrm{e}-12$ & $9.90 \mathrm{e}-11$ & 0.998 & $8.20 \mathrm{e}-01$ & $4.09 \mathrm{e}+04$ \\
0.1 & 0.1 & $1 \mathrm{e}-11$ & $9.09 \mathrm{e}-10$ & 0.982 & $8.34 \mathrm{e}-01$ & $4.85 \mathrm{e}+03$ \\
0.5 & 0.1 & $5 \mathrm{e}-11$ & $3.33 \mathrm{e}-09$ & 0.936 & $8.75 \mathrm{e}-01$ & $1.80 \mathrm{e}+03$ \\
0.9 & 0.1 & $9 \mathrm{e}-11$ & $4.74 \mathrm{e}-09$ & 0.910 & $9.00 \mathrm{e}-01$ & $1.61 \mathrm{e}+03$ \\
0.99 & 0.1 & $9.9 \mathrm{e}-11$ & $4.97 \mathrm{e}-09$ & 0.905 & $9.04 \mathrm{e}-01$ & $1.60 \mathrm{e}+03$ \\
\hline \hline
\end{tabular}

TABLE I

TABULATED NUMERICAL VALUES FOR PARAMETERS.

Since $P_{1}$ and $P_{2}$ are linearly independent, the scalars $\alpha_{1}$ and $\alpha_{1}$ can be uniquely solved (see Appendix C). We can now rewrite (40) in terms of $\alpha_{1}, \alpha_{2}, P_{1}, P_{2}$ as:

$$
V_{C_{s} m}=V-\alpha_{1} A R^{m} P_{1}-\alpha_{2} A R^{m} P_{2} .
$$

By definition, $R^{m} P_{1}=\lambda_{1}^{m} P_{1}$ and $R^{m} P_{2}=\lambda_{2}^{m} P_{2}$ :

$$
V_{C_{s} m}=V-\alpha_{1} \lambda_{1}^{m} A P_{1}-\alpha_{2} \lambda_{2}^{m} A P_{2} .
$$

We define the scalar constants $k_{1}=\alpha_{1} A P_{1}$ and $k_{2}=\alpha_{2} A P_{2}$; they are computed as:

$$
\begin{gathered}
k_{1}=\alpha_{1} A P_{1}=\frac{(2-a-b+c)}{2 c} V, \\
k_{2}=\alpha_{2} A P_{2}=-\frac{(2-a-b-c)}{2 c} V,
\end{gathered}
$$

where

$$
c=\sqrt{(2-a)^{2}+(2-b)^{2}-2 a b-4} .
$$

Finally, (38) becomes:

$$
V_{C_{s} m}=V-k_{1} \lambda_{1}^{m}-k_{2} \lambda_{2}^{m} .
$$

Table I shows calculated values for $\lambda_{1}, \lambda_{2}, k_{1}$ and $k_{2}$ for various ratios of $C_{c} / C_{s}$ and $t_{0} / \tau$. One eigenvalue is often larger than the other, e.g. $\lambda_{1}>\lambda_{2}$. In our case, this is especially true when $C_{s}>C_{c}$. When the eigenvalues are raised to the power $m$, for large values of $m$ the difference between the eigenvalues grows, such that the larger eigenvalue, $\lambda_{1}$, dominates. Thus, when a matrix, raised to a large power, is applied to a vector which is a linear combination of its eigenvectors, the result can be approximated by the eigenvector of the corresponding dominant eigenvalue, multiplied by the eigenvalue raised to the same large power. Table I shows that, especially when $C_{s} \gg C_{c} ; k_{1}>k_{2}$ and $\lambda_{1}>\lambda_{2}$, and often they are larger by orders of magnitude. The dominance of $k_{1}$ and $\lambda_{1}$, together with the power of $\lambda$, lets us approximate (53) as:

$$
V_{C_{s} m} \approx V-k_{1} \lambda_{1}^{m} \text {, }
$$




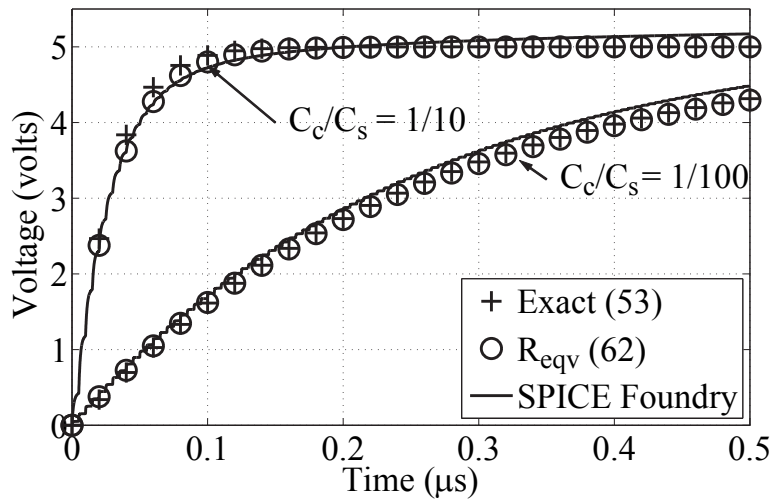

Fig. 4. Comparison of the exact solution, (53), $R_{\text {eqv }}$, (62), and a SPICE simulation using a foundry diode. $C_{s}=100 \mathrm{pF}, R_{s}=100 \Omega, f=100 \mathrm{M} \mathrm{Hz}$

and, using (41),

$$
\Delta V_{m} \approx k_{1} \lambda_{1}^{m} .
$$

\section{C. $R_{\text {eqv }}$ derivation}

With a simple representation of $V_{C_{s} m}$ we now seek to find $i_{a v g}$ and $R_{e q v}$. We start with the charge delivered in one step,

$$
\begin{aligned}
Q_{m} & =C_{s}\left(V_{C_{s} m+1}-V_{C_{s} m}\right), \\
Q_{m} & =C_{s}\left(V-k_{1} \lambda_{1}^{m+1}-V+k_{1} \lambda_{1}^{m}\right), \\
Q_{m} & =C_{s} k_{1} \lambda_{1}^{m}\left(1-\lambda_{1}\right) .
\end{aligned}
$$

and

$$
i_{\text {avg }, m}=\frac{Q_{m}}{2 t_{0}}=\frac{C_{s} k_{1}\left(\lambda_{1}\right)^{m}\left(1-\lambda_{1}\right)}{2 t_{0}} .
$$

$R_{e q v}$ can now be calculated:

$$
\begin{aligned}
R_{\text {eqv }} & =\frac{\Delta V_{m}}{i_{\text {avg }, m}}, \\
R_{\text {eqv }} & \approx \frac{2 t_{0} k_{1}\left(\lambda_{1}\right)^{m}}{C_{s} k_{1}\left(\lambda_{1}\right)^{m}\left(1-\lambda_{1}\right)}, \\
R_{\text {eqv }} & \approx \frac{2 t_{0}}{C_{s}\left(1-\lambda_{1}\right)} .
\end{aligned}
$$

We now have in (62) a single parameter $R_{\text {eqv }}$ that lets a designer model the entire capacitive transfer system as shown in Fig. 2(b). Fig. 4 compares the capacitor voltage $V_{C_{s}}$ for the exact analytical solution (53), solution based on the $R_{\text {eqv }}$ model, (62), and a SPICE simulation of a foundry model. It can be seen that the $R_{e q v}$ model is very good.

\section{Model Limits}

In the previous subsection we have derived an equivalent resistance $\left(R_{\text {eqv }}\right)$ model for the switching source, coupling capacitors and nonlinear rectifier and $R_{s} \neq 0,(62)$. Here we examine the limits of this model as $f \rightarrow \infty$ and $R_{s} \rightarrow 0$.

We begin with the case of $R_{s} \neq 0, R_{e q v}$, (62), is

$$
R_{e q v}=\frac{2 t_{0}}{C_{s}\left(1-\lambda_{1}\right)} .
$$

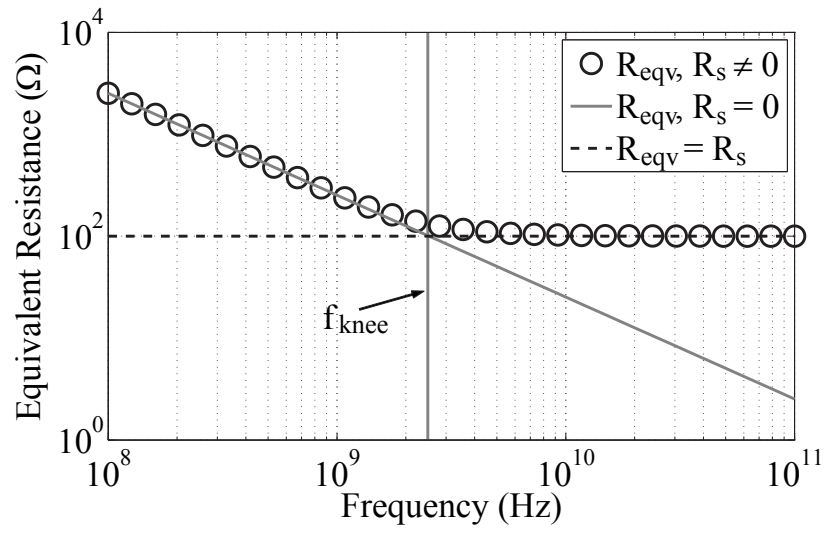

Fig. 5. $R_{e q v}$ versus frequency. As frequency increases, $R_{e q v} \rightarrow R_{s}$. $C_{s}=$ $100 p F, C_{c}=1 p F, R_{s}=100 \Omega$.

As $t_{0} \rightarrow 0$, or $f \rightarrow \infty, R_{e q v}$ approaches to:

$$
\lim _{t_{0} \rightarrow 0} R_{e q v}=\lim _{f \rightarrow \infty} R_{e q v}=\frac{0}{0},
$$

which is indeterminate. By using L'Hôspital's Rule we find $\lim _{t_{0} \rightarrow 0} R_{e q v}$ as:

$$
\lim _{t_{0} \rightarrow 0} R_{e q v}=R_{s} .
$$

This result says that as frequency increases, the added effective impedance of the rectifier and coupling capacitors goes to zero, and at $f=\infty$, the only resistance element in the equivalent model is the lumped series resistance of the circuit. Fig. 5 plots $R_{e q v}$ for a given set of parameters versus frequency. At low frequencies the coupling capacitors and rectifying bridge dominate the $R_{\text {eqv }}$. Also plotted is $R_{\text {eqv }}$ for $R_{s}=0$; while the series resistance is negligible, the effective impedance of the coupling capacitors and rectifier create a significant $R_{e q v}$. In fact, one can see that at low frequencies $R_{e q v}$ is almost identical for both the $R_{s}=0$ and $R_{s} \neq 0$, which makes sense if in both cases the dominant effective impedance does not come from the series resistance, $R_{s}$, but rather the finite delivery time of charge due to a low frequency of charging. At higher frequencies, the limitation of charge delivery due to the frequency reduces and $R_{s}$ dominates.

We now consider the case $R_{s} \neq 0$, (62), when $R_{s} \rightarrow 0$. In this limit $R_{\text {eqv }}$ becomes

$$
\lim _{R_{s} \rightarrow 0} R_{e q v}=\frac{t_{0}\left(C_{c}+C_{s}\right)}{2 C_{s} C_{c}} .
$$

This is shown in Fig. 5 as $R_{e q v}$ reduces with frequency with a slope of -1 . This result, (66), is the same that is found by using a switch-capacitor approach (Appendix A) and demonstrates that the $R_{\text {eqv }}$ for $R_{s} \neq 0$ is actual a general expression for all $R_{s}$ or $\tau$.

Finally we define the critical frequency, $f_{k n e e}$, as the crossover point of the two asymptotes in Fig. 5. The critical frequency can be easily calculated by intersecting the line equations:

$$
R_{\text {eqv }, \text { knee }}=\frac{1}{2 f_{\text {knee }}} \frac{\left(C_{c}+C_{s}\right)}{2 C_{s} C_{c}}=R_{s} .
$$



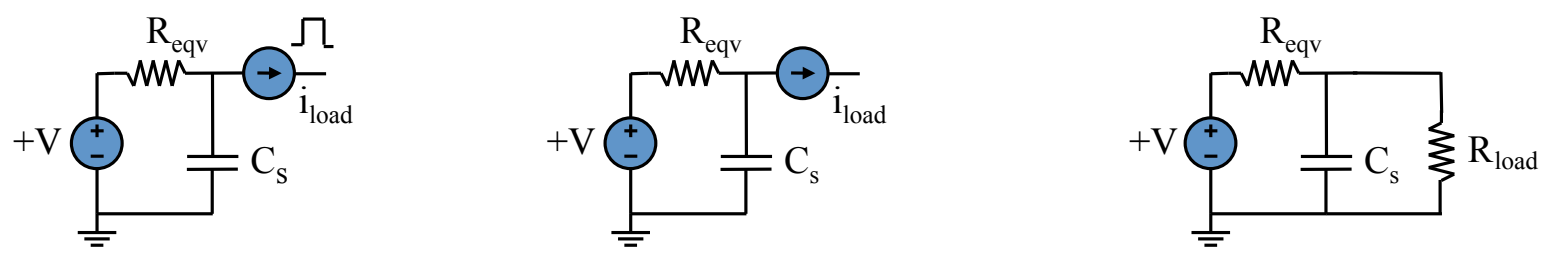

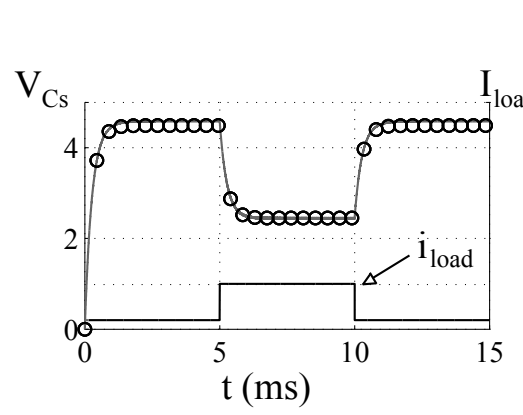

(a)
- SPICE Foundry

O Based on $R_{\text {eqv }}(62)$

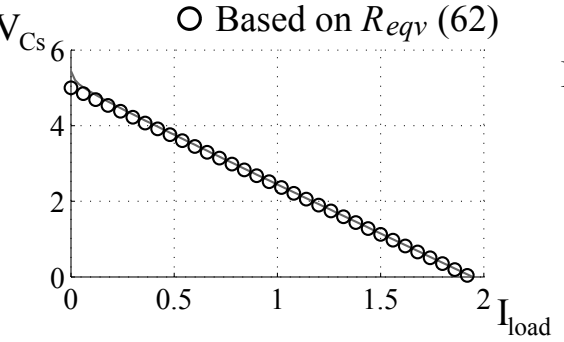

(b)

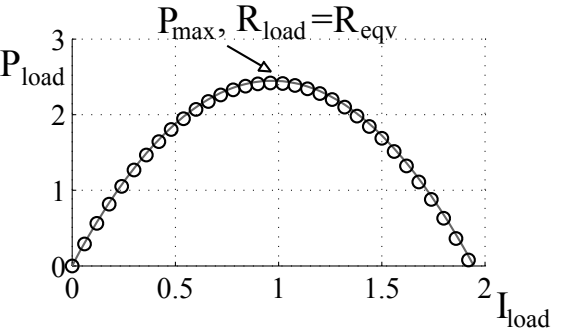

(c)

Fig. 6. Example application using $R_{e q v}$ (a) Equivalent model transient behavior. (b) Equivalent model load line. (c) Equivalent model maximum power versus Iload.

$$
f_{\text {knee }}=\frac{\left(C_{c}+C_{s}\right)}{4 R_{s} C_{s} C_{c}}=\frac{1}{4 \tau} .
$$

It is not surprising that the resulting critical frequency $f_{k n e e}$ is a simple function of the circuit's time constant $\tau$.

\section{IV. $R_{e q v}$ ApPlication AND Design CRiteria}

In this section we demonstrate the application of the $R_{e q v}$ model through comparison to simulations using a foundry model and also examine the limits of $R_{e q v}$ as the circuit parameters are varied. These show the usefulness of the model and also how the designer can easily explore the design space for capacitive power transfer with the generalized $R_{e q v}$ model developed in this work.

\section{A. $R_{e q v}$ as a Model for Capacitive Power Delivery.}

The $R_{e q v}$ model developed in the previous section allows the designer to replace the entire rectifier circuit with a simply resistor circuit. Figure 6 shows several simple applications of the model and compares them to SPICE simulations using a foundry diode. Figure 6(a) shows the transient output voltage in response to an input step voltage. Both the droop as well as the transient waveform are modeled well by $R_{q e q v}$. Figure 6(b) shows the $d c$ load-line for a steady-state current load. This allows a designer to determine the output voltage for a given load or more importantly, to determine the needed $R_{e q v}$, and with it the needed $R_{s}, f$ and $C_{c} / C_{s}$, for a given design goal. Figure 6(c) shows the power delivered to a load for a varying load currents. As expected, the maximum power transfer occurs when $R_{\text {load }}=R_{\text {eqv }}$, where $R_{\text {load }}$ can be derived from $I_{\text {load }}$ and the loadline in Fig. 6(b). One can see that $R_{e q v}$ models the rectifier dynamics very well.

\section{B. $R_{\text {eqv }}$ Design Space}

In designing capacitive power transfer systems using the $R_{e q v}$ model, it is helpful to see the dependence of the model on the circuit parameters. To show this, we note that in (62) $R_{e q v}$ is proportional to $1 /\left(1-\lambda_{1}\right)$. Figure 7 (a) plots $1 /\left(1-\lambda_{1}\right)$ versus $f \cdot R_{s}$ for several capacitors ratios $C_{c} / C_{s}$. We plot versus $f \cdot R_{s}$ as $1 /\left(1-\lambda_{1}\right)$ varies in the same manner with an increase in $f$ or $R_{s}$, showing the interplay between the time constant determined by $R_{s}$ and the charge cycle time, $t_{0}$ or $1 / f$. Figure 7 (a) shows two regions of operation. In region I, $R_{e q v}$ is independent of $f \cdot R_{s}$ and is determined by the capacitor ratio alone; in this region reducing $R_{s}$ will not reduce $R_{e q v}$ as $\tau<t_{0}$, i.e. the capacitors fully charge each cycle. In region II, $R_{e q v}$ is determined by $f \cdot R_{s}$; in this region $\tau \geq t_{0}$ and power transfer is limited by $\tau$, which is a function of the series resistance and capacitance values, and the charge cycle period. Figure 7(b) shows the dependence of $1 /\left(1-\lambda_{1}\right)$ on the capacitor ratios, $C_{c} / C_{s}$, for a series of $f \cdot R s$ values. We show again where in Region $1, R_{e q v}$ is limited by the capacitor ratio and in Region 2 we are limited by $f \cdot R_{s}$. In both of these we see that the key design parameters are $f \cdot R_{s}$ and $C_{c} / C_{s}$.

The separation of the regions can be determined by examining $1 /\left(1-\lambda_{1}\right)$ for small and large $f \cdot R_{s}$. In Region 1 , as $f \cdot R_{s}$ approaches zero, the value of $1 /\left(1-\lambda_{1}\right)$ becomes

$$
\frac{1}{\left(1-\lambda_{1}\right)} \simeq \frac{\left(C_{c}+C_{s}\right)^{2}}{4 C_{c} C_{s}},
$$

which represents the horizontal trajectories of Region 1 in Fig.7(a).

When $f \cdot R_{s}$ is very large, it becomes

$$
\frac{1}{\left(1-\lambda_{1}\right)} \simeq f \cdot R_{s} C_{s}
$$

which represents the diagonal line in Region 2 in Fig.7(a).

Using these two equations, we can solve for the crossover between regions 1 and 2:

$$
\left(f \cdot R_{s}\right)_{k n e e}=\frac{1}{4 C_{c}}\left(1+\frac{C_{c}}{C_{s}}\right)^{2} .
$$




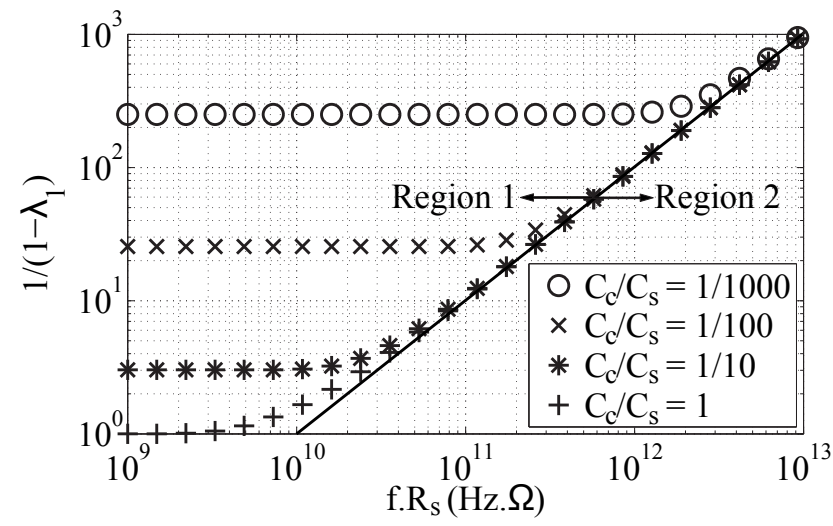

(a)

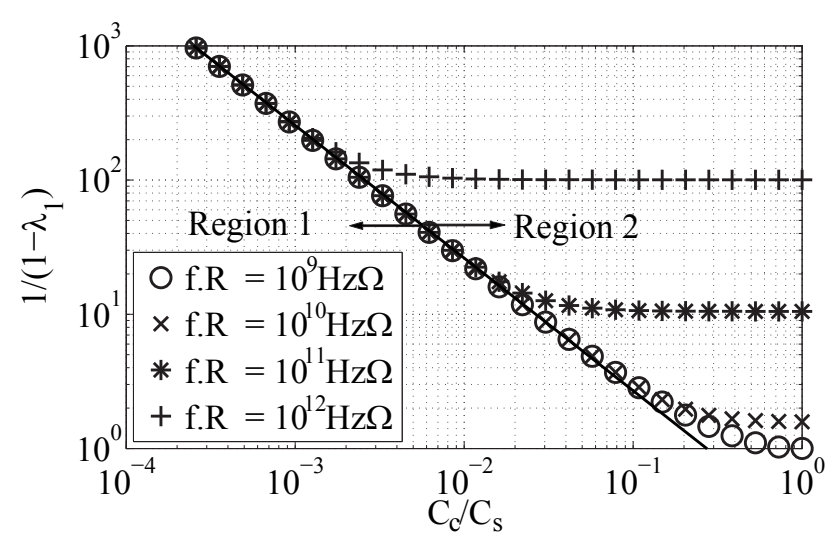

(b)

Fig. 7. (a) Plot of $1 /\left(1-\lambda_{1}\right)$ versus $f \cdot R_{s}$. (b) Plot of $1 /\left(1-\lambda_{1}\right)$ versus $C_{c} / C_{s}$. Two regions of operation are seen: Region 1 is dominated by $C_{c} / C_{s}$. Region 2 is dominated by $f \cdot R_{s}$.

We can do a similar analysis for the curve in Fig.7(b). As the ratio $C_{c} / C_{s}$ approaches 1 (from the left), the value of $1 /\left(1-\lambda_{1}\right)$ becomes

$$
\frac{1}{\left(1-\lambda_{1}\right)} \simeq \frac{1}{1-e^{-1 /\left(f C_{s} R_{s}\right)}}
$$

Similarly, when $C_{c} / C_{s}$ approaches $0,1 /\left(1-\lambda_{1}\right)$ becomes

$$
\frac{1}{\left(1-\lambda_{1}\right)} \simeq \frac{1}{\left(C_{c} / C_{s}\right)} \frac{1}{4}
$$

Again, we can find the knee:

$$
\left(\frac{C_{c}}{C_{s}}\right)_{k n e e}=\frac{1}{4}\left(1-e^{-1 /\left(f C_{s} R_{s}\right)}\right) .
$$

\section{Power Considerations}

In addition to designing load-line and transient dynamics with $R_{e q v}$, it is possible to model the power dissipation of the rectifier with $R_{e q v}$. This is extremely useful, as a detailed power calculation of a bridge rectifier can be tedious. Figure 8 plots the power dissipation calculated using the actual instantaneous currents through the rectifier and the equivalent power dissipation if one used $R_{e q v}$, (62). For $C_{c} \ll C_{s}$, they match very well. This can be understood from an energy argument. Each electron that leaves the source looses $q \Delta V$ of energy due to $R_{e q v}$. In one cycle, the total charge is $i_{a v g} \cdot t_{0}$. If we assume that $\Delta V$ does not change significantly during a cycle, i.e. $\Delta V\left(t \rightarrow t_{0}\right) \approx \Delta V_{m}$, then the energy lost, or work done, due to $R_{\text {eqv }}$ in one cycle is

$$
\begin{gathered}
W_{\text {eqv }}=q \Delta V=i_{\text {avg }} \cdot t_{0} \cdot \Delta V_{m}, \\
P_{\text {dissipated }}=\frac{W_{\text {eqv }}}{t_{0}}=i_{\text {avg }} \cdot \Delta V_{m},
\end{gathered}
$$

and

$$
\begin{aligned}
R_{e q v} & \equiv \frac{\Delta V}{i_{a v g}}, \\
i_{\text {avg }} & =\frac{R_{e q v}}{\Delta V},
\end{aligned}
$$

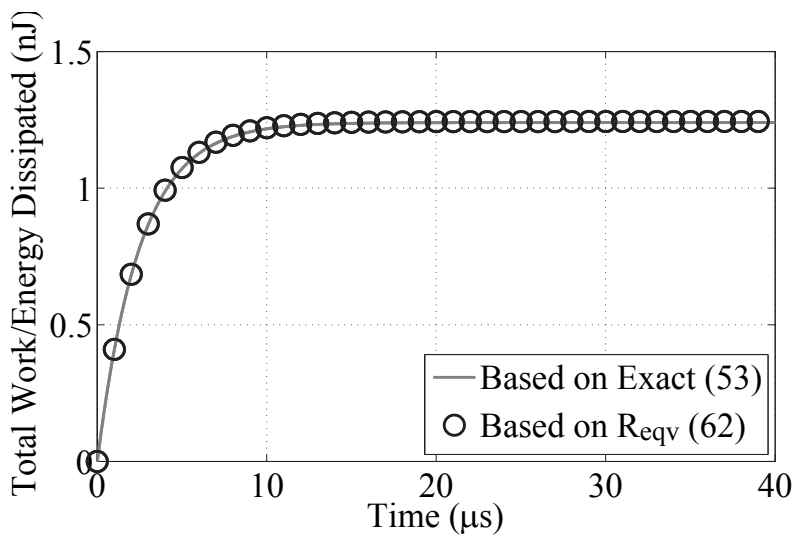

Fig. 8. Cumulative energy dissipation using the exact solution (53) and $R_{\text {eqv }}$ based model, (62). $C_{c}=0.1 p F, C_{s}=100 p F, R_{s}=1000 \Omega$, $f=100 M H z$.

resulting in

$$
P_{\text {dissipated }}=P_{R_{\text {eqv }}}=\frac{\Delta V^{2}}{R_{\text {eqv }}} .
$$

\section{CONCLUSION}

In this paper we have developed a simple model for capacitive power transfer through an equivalent $R C$ model: $R_{e q v} \cdot C_{s}$. We accomplished this by using a power iteration method to solve the coupled equations for all time constants, $\tau$. These analytical solutions were then used to to calculate an equivalent resistance model, $R_{e q v}$. With this model developed, we applied it to steady-state and transient load conditions and showed that it is a very accurate model. In addition, we showed that energy dissipation of the rectifying stage can be modeled with $R_{e q v}$. Finally, we used the model to examine the design space for capacitive power transfer, in particular the regimes where different circuit parameters dominate.

\section{ACKNOWLEDGMENTS}

We acknowledge the support of Intel Research Pittsburgh. We also thank Xin Li and Rob Reid for helpful discussions. 
APPENDIX A

CASE $C_{c}<C_{s} ; \tau \ll t_{0}$

We analyze the case when the effective series resistance, and thus the effective time constant of the coupling circuit, is substantially small compared to the source half period $t_{0}$. This allows us to approximate the term $i(t) R_{s}$ as 0 just before the end of each source voltage half period, $t=n t_{0}^{-}$. This is the same as assuming $t_{0}$ is large enough that the capacitors are fully charged before the next source voltage transition. With the assumption that at the end of each cycle, $i(t) R_{s} \approx 0$, (1) and (11) become:

$$
\begin{gathered}
V-V_{C_{s} n}-V_{C_{c} n}=0, \quad n=\text { odd }, \\
-V+V_{C_{s} n}-V_{C_{c} n}=0, \quad n=\text { even },
\end{gathered}
$$

Equations (6) and (14) can also be written as:

$$
\begin{gathered}
V_{C_{s} n}=a_{0} V+\left(1-a_{0}\right) V_{C_{s}(n-1)}-a_{0} V_{C_{c}(n-1)}, n=\text { odd }, \\
V_{C_{s} n}=a_{0} V+\left(1-a_{0}\right) V_{C_{s}(n-1)}+a_{0} V_{C_{c}(n-1)}, n=\text { even. }
\end{gathered}
$$

where $a_{0}$ and $b_{0}$ are constants derived from (8) and (9):

$$
\begin{aligned}
& a_{0}=\lim _{\tau \rightarrow 0} a(t)=\frac{C_{s}}{C_{c}+C_{s}}, \\
& b_{0}=\lim _{\tau \rightarrow 0} a(t)=\frac{C_{s}}{C_{c}+C_{s}} .
\end{aligned}
$$

Using (80) and (81) to solve for $V_{C_{s} n}$ in terms of $V_{C_{c} n}$, (82) and (83) can be combined:

$$
\begin{aligned}
V_{C_{s} n}= & a_{0} V+\left(1-a_{0}\right) V_{C_{s}(n-1)} \\
& -a_{0}\left(-V+V_{C_{s}(n-1)}\right) \\
= & 2 a_{0} V+\left(1-2 a_{0}\right) V_{C_{s}(n-1)} \\
= & \frac{2 C_{c}}{C_{c}+C_{s}} V+\frac{C_{s}-C_{c}}{C_{c}+C_{s}} V_{C_{s}(n-1)} .
\end{aligned}
$$

Equation (88) is valid for $n \geq 2$.

The series can be simplified to yield:

$$
V_{C_{s} n}=V\left[1-\left(\frac{C_{s}-C_{c}}{C_{c}+C_{s}}\right)^{n-1}\right]+V_{C_{s} 1}\left(\frac{C_{s}-C_{c}}{C_{c}+C_{s}}\right)^{n-1} .
$$

With the solution of the voltage on the storage capacitor, $V_{C_{s} n}$, we can now proceed with the procedure outlined in Sec. II.

We begin by solving for the charge transferred in a cycle:

$$
\begin{aligned}
Q_{n} & =C_{s}\left(V_{C_{s} n+1}-V_{C_{s} n}\right), \\
& =V C_{s} \frac{C_{s}}{C_{s}-C_{c}}\left(\frac{C_{s}-C_{c}}{C_{c}+C_{s}}\right)^{n}\left(1-\frac{C_{s}-C_{c}}{C_{c}+C_{s}}\right) .
\end{aligned}
$$

We define the average current during each cycle as:

$$
i_{a v g, n}=\frac{Q_{n}}{t_{0}}
$$

and substitute in $Q_{n}$, (91),

$$
i_{a v g, n}=\frac{V C_{s}}{t_{0}} \frac{C_{s}}{C_{s}-C_{c}}\left(\frac{C_{s}-C_{c}}{C_{c}+C_{s}}\right)^{n}\left(\frac{2 C_{c}}{C_{c}+C_{s}}\right) .
$$

We now find $\Delta V_{n} / i_{\text {avg,n }}$ using (93) and by calculating $\Delta V_{n}$ from (89):

$$
\frac{\Delta V_{n}}{i_{a v g, n}}=\frac{t_{0}}{C_{s}\left(\frac{2 C_{c}}{C_{c}+C_{s}}\right)}
$$

and equivalent resistance, $R_{e q v}$, is:

$$
R_{e q v}=\frac{t_{0}\left(C_{c}+C_{s}\right)}{2 C_{s} C_{c}}
$$

\section{APPENDIX B}

EIGENVALUES AND EIGENVECTORS OF $R$

The eignevalues of $\mathrm{R}$ are given below:

$$
\begin{aligned}
\lambda_{1}= & \frac{1}{2}\left((1-a)^{2}+(1-b)^{2}-2 a b\right. \\
& \left.+(b-a) \sqrt{(2-a)^{2}+(2-b)^{2}-2 a b-4}\right), \\
\lambda_{2}= & \frac{1}{2}\left((1-a)^{2}+(1-b)^{2}-2 a b\right. \\
& \left.-(b-a) \sqrt{(2-a)^{2}+(2-b)^{2}-2 a b-4}\right) .
\end{aligned}
$$

The eigenvectors of $\mathrm{R}$ are given below:

$$
\begin{aligned}
& P_{1}=\left[\begin{array}{c}
\frac{2-a-b+c}{2 b} \\
1
\end{array}\right] . \\
& P_{2}=\left[\begin{array}{c}
\frac{2-a-b-c}{2 b} \\
1
\end{array}\right] .
\end{aligned}
$$

\section{APPENDIX C}

\section{REPRESENTATION OF $W$ IN EIGENVECTORS OF $R$}

$$
W=\alpha_{1} P_{1}+\alpha_{2} P_{2}
$$

where:

$$
\begin{array}{r}
\alpha_{1}=(a-b) b V \\
-\frac{b\left(a(2-a+b)+\frac{1}{2}(a-b)(-2+a+b+c)\right)}{c} V, \\
\alpha_{2}=\frac{b\left(a(2-a+b)+\frac{1}{2}(a-b)(-2+a+b+c)\right)}{c} V .
\end{array}
$$

\section{REFERENCES}

[1] A. Sodagar and P. Amiri, "Capacitive coupling for power and data telemetry to implantable biomedical microsystems," in Neural Engineering, 2009. NER '09. 4th International IEEE/EMBS Conference on, 29 2009may 2 2009, pp. $411-414$.

[2] K. A. Cook-Chennault, N. Thambi, and A. M. Sastry, "Powering mems portable devices - a review of non-regenerative and regenerative power supply systems with special emphasis on piezoelectric energy harvesting systems," Smart Materials and Structures, vol. 17, no. 4, p. 043001, 2008.

[3] S. Hollar, A. Flynn, C. Bellew, and K. Pister, "Solar powered $10 \mathrm{mg}$ silicon robot," Micro Electro Mechanical Systems, 2003. MEMS-03 Kyoto. IEEE The Sixteenth Annual International Conference on, pp. 706-711, Jan. 2003.

[4] M. Makowski and D. Maksimovic, "Performance limits of switchedcapacitor dc-dc converters," in Power Electronics Specialists Conference, 1995. PESC '95 Record., 26th Annual IEEE, vol. 2, jun 1995, pp. 1215 -1221 vol.2. 
[5] G. Cataldo and G. Palumbo, "Double and triple charge pump for power ic: dynamic models which take parasitic effects into account," Circuits and Systems I: Fundamental Theory and Applications, IEEE Transactions on, vol. 40, no. 2, pp. 92 -101, feb 1993.

[6] T. Tanzawa and T. Tanaka, "A dynamic analysis of the dickson charge pump circuit," Solid-State Circuits, IEEE Journal of, vol. 32, no. 8, pp. $1231-1240$, aug 1997.

[7] M. E. Karagozler, S. C. Goldstein, and J. R. Reid, "Stress-driven mems assembly + electrostatic forces $=1 \mathrm{~mm}$ diameter robot." in Proceedings of the IEEE International Conference on Intelligent Robots and Systems (IROS '09)., 2009.

[8] R. Duggirala, H. Li, and A. Lal, "Active circuits for ultra-high efficiency micropower generators using nickel-63 radioisotope," in Solid-State Circuits Conference, 2006. ISSCC 2006. Digest of Technical Papers. IEEE International, feb. 2006, pp. $1648-1655$.

[9] S. O'Driscoll, A. Poon, and T. Meng, "A mm-sized implantable power receiver with adaptive link compensation," in Solid-State Circuits Conference - Digest of Technical Papers, 2009. ISSCC 2009. IEEE International, feb. 2009 , pp. $294-295,295$ a.

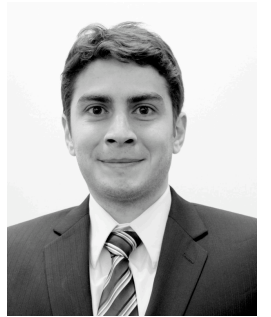

Mustafa Emre Karagozler received the B.S. degree in Electrical and Electronics Engineering from Middle East Technical University, Turkey, in 2004 and the M.S. degree in Electrical and Computer Engineering from Carnegie Mellon University in 2007.

$\mathrm{He}$ is currently working toward the Ph.D. degree in the Electrical and Computer Engineering at Carnegie Mellon University. His Ph.D. research focuses on how to make and use programmable matter. He currently investigates the use of forceat-a-distance effectors as mechanisms to actuate microrobots.

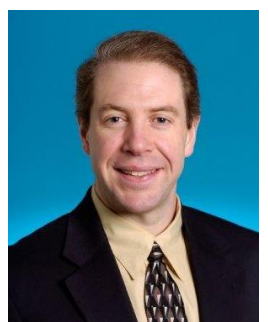

David S. Ricketts received the $\mathrm{PhD}$ in Electrical Engineering from Harvard University in 2006 and the B.S. (1995) and M.S. (1997) degrees in Electrical Engineering from Worcester Polytechnic Institute (WPI). He is currently an Assistant Professor of Electrical and Computer Engineering at Carnegie Mellon University and is also a courtesy faculty in the Material Science and Engineering department. $\mathrm{He}$ has more than 8 years industrial experience in the development of $40+$ integrated circuits in mixed-signal, RF and power management applications. Prof. Ricketts research crosses the fields of physics, material science and circuit design, investigating the ultimate capabilities of microelectronic devices and how these are harnessed by differing circuit topologies to produce the highest performing systems. His work has appeared in Nature and in numerous IEEE conferences and journals and was selected for the 2008 McGraw Hill Yearbook of Science and Engineering. He is the author of the two books on jitter in high-speed electronics and electrical solitons. He is the recipient of the NSF CAREER Award, the DARPA Young Faculty Award and the George Tallman Ladd research award and was a Harvard Innovation Fellow and 2009 Wimmer Faculty Teaching Fellow.

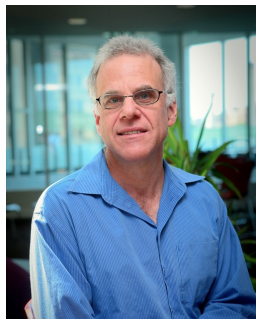

Seth Copen Goldstein (M'96-SM'06) received the E.E.C.S degree in 1985 from Princeton University and M.S. and Ph.D. degrees in computer science from the University of California, Berkeley, in 1994 and 1997, respectively.

$\mathrm{He}$ is currently an Associate Professor in the School of Computer Science, Carnegie Mellon University, Pittsburgh, PA. His current research interests include large collections of interacting agents. In the area of reconfigurable computing, he investigated how to compile high-level programming languages directly into configurations that could harness the large ensemble of gates for computing. Later work involved ensembles of molecules in the area of molecular electronics. This research investigated how to design, manufacture, and use molecular-scale devices for computing. He is currently involved in realizing Claytronics, a form of programmable matter. 